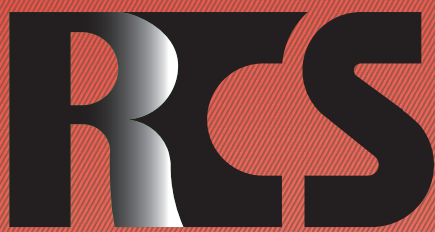

Depósito legal ppi $201502 Z U 4662$

Esta publicación científica en formato digital es continuidad de la revista impresa Depósito Legal: pp $197402 Z$ U789

- ISSN: 1315-9518 • ISSN-E: 2477-9431

Revista de Ciencias Sociales

Universidad del Zulia. Revista de la Facultad de Ciencias Económicas y Sociales Vol. XXVII,

No. 4,2021

Esta publicación científica en formato digital es continuidad de la revista impresa Depósito Legal: pp $197402 Z U 789$ ISSN: $1315-9518$ 


\title{
Brecha de género en la graduación de ingenieras industriales peruanas
}

\author{
Ruiz-Ruiz, Marcos Fernando" \\ Noriega-Aranibar, María Teresa ${ }^{* *}$ \\ Pease-Dreibelbis, María Angélica***
}

\begin{abstract}
Resumen
En las últimas décadas la presencia femenina ha aumentado en áreas vinculadas a las ciencias e ingenierías. No obstante, su participación sigue siendo incipiente en muchos de esos campos. En consecuencia, el objetivo de este estudio fue explicar cómo un grupo de profesoras ingenieras representan las vivencias de sus alumnas en un contexto caracterizado por el dominio masculino. La metodología fue exploratoria y descriptiva, entrevistando a 24 profesoras peruanas. Los hallazgos identifican que las estudiantes exhiben conductas y participación diferenciadas respecto a los varones, asociadas a expectativas de desempeño, atribución de características y trato diferenciado por parte de los docentes que tienden a disminuirlas respecto a los varones. Además, tienden a mayores cargas familiares afectando su permanencia en los estudios. Ello, sin embargo, varía en función de la universidad, siendo la situación de las mujeres de universidades públicas la más vulnerable. El estudio concluye que las entrevistadas muestran una visión crítica de estas situaciones, pero con tendencia a sobrevalorar las capacidades femeninas y al desarrollo de ciertos atributos para conquistar el mercado laboral; antes que a eliminar las barreras que las excluyen del mismo. Esta exigente realidad para las jóvenes explicaría su baja representación en la ingeniería industrial peruana.
\end{abstract}

Palabras clave: Género; ingeniería industrial; igualdad de oportunidades; educación superior; Perú.

\footnotetext{
* Doctor en Ciencias de la Educación. Ingeniero Industrial. Profesor Titular e Investigador de la Facultad de Ingeniería y Arquitectura de la Universidad de Lima, Perú. E-mail: mruiz@ulima.edu.pe

(iD) ORCID: https://orcid.org/0000-0001-5147-8512

** Doctora en Ciencias Contables y Empresariales. Ingeniera industrial. Profesora Titular e Investigadora de la Facultad de Ingeniería y Arquitectura de la Universidad de Lima, Perú. E-mail: manorieg@ulima. edu.pe (iD) ORCID: https://orcid.org/0000-0001-6824-1415

*** Doctora en Psicología Cognitiva y Antropóloga. Profesora Titular e Investigadora del Departamento Académico de Psicología de la Pontificia Universidad Católica del Perú, Perú.. E-mail: mapease@ pucp.edu.pe (iD ORCID: https://orcid.org/0000-0003-2645-4580
} 


\title{
Gender gap in the graduation of Peruvian industrial engineers
}

\begin{abstract}
In recent decades, the female presence has increased in areas related to science and engineering. However, its participation is still incipient in many of these fields. Consequently, the objective of this study was to explain how a group of female engineering teachers represent the experiences of their students in a context characterized by male dominance. The methodology was exploratory and descriptive, interviewing 24 Peruvian teachers. The findings identify that female students exhibit differentiated behaviors and participation with respect to males, associated with performance expectations, attribution of characteristics and differentiated treatment by teachers that tend to decrease them with respect to males. In addition, they tend to have greater family burdens, affecting their permanence in studies. This, however, varies depending on the university, with the situation of women from public universities being the most vulnerable. The study concludes that the interviewees show a critical vision of these situations, but with a tendency to overvalue female capacities and to develop certain attributes to conquer the labor market; rather than to eliminate the barriers that exclude them from it. This demanding reality for young women would explain their low representation in Peruvian industrial engineering
\end{abstract}

Keywords: Gender; industrial engineering; equal opportunities; higher education; Peru.

\section{Introducción}

La importancia de la graduación y desarrollo de profesionales en las áreas STEM (ciencia, tecnología, ingeniería y matemáticas) son claves para el progreso de las sociedades, toda vez que permiten la formación de individuos y colaboradores orientados a la solución de diversos problemas tecnológicos y sociales; así como con las competencias para adaptarse a las exigencias del mercado (Reinking y Martin, 2018; Barragán, González y Ortiz, 2020). En este ámbito, diversos investigadores se han centrado en estudiar las diferencias asociadas con el género y la reducida participación de las mujeres en las áreas STEM.

En el caso de las ingenierías, si bien la participación femenina ha ido aumentando en las últimas décadas y, por consiguiente, se han advertido avances en la reducción de la mencionada brecha de género en países como Estados Unidos; tal participación sigue siendo todavía incipiente en muchas de sus subespecialidades (Sarathchandra et al., 2018). Para el caso específico de la ingeniería industrial, el panorama no es diferente. Esta subespecialidad de la ingeniería se centra, como lo indica el Institute of Industrial \& Systems Engineers (IISE, 2019), en el diseño, mejoramiento e instalación de sistemas integrados de personas, equipos, materiales y energía. Se orienta, además, a la adquisición de conocimientos y habilidades especializadas en las ciencias matemáticas, físicas, así como sociales -junto con los principios y métodos del análisis y diseño- para especificar, predecir y evaluar los resultados que se obtendrán en los sistemas anteriormente especificados. Por su parte, algunos autores sugieren que esta rama de la ingeniería se caracteriza por un fuerte componente social, reflejado en su mayor calidez, flexibilidad y lo que llaman "sentido femenino" (Faulkner, 2009a; 2009b; Brawner et al., 2012).

Resulta paradójico, frente a estas características que podrían resultar atractivas tanto a hombres como mujeres, evidenciar 
que la participación femenina en la ingeniería industrial esté reducida en países como el Perú, respectivamente, a una postulación y graduación de $36,4 \%$ y $33 \%$ durante el año 2017, según la Superintendencia Nacional de Educación Superior Universitaria del Perú (SUNEDU, 2019).

Esta realidad resulta particularmente interesante en la medida que otras subespecialidades de la ingeniería, tales como la ingeniería ambiental y la ingeniería química, textil y afines, reportaron para el mismo año una tasa de participación femenina en su graduación de 50,9\% y 53,2\%, respectivamente. Es decir, una mayor participación femenina que evidencia que las ingenierías no son, necesariamente, un bastión de dominio masculino en todas sus subespecialidades. Por el contrario, hay razones para ir quebrando aquellos estereotipos de género que refuerzan la idea que la ingeniería es apropiada sólo para los hombres y que las mujeres que entran en estas áreas deben probar continuamente su idoneidad como profesionales (Dryburgh, 1999).

Frentea la realidadanteriormente descrita y contextualizada en un país sudamericano como Perú, donde la investigación en torno a la participación femenina en las áreas STEM es prácticamente inexistente, se propone explorar en torno a las razones asociadas a la baja representación femenina en la graduación de ingenieras industriales peruanas. Para ello, se decide abordar el problema de investigación, estrictamente desde la percepción de las mismas ingenieras industriales que ejercen la docencia en algunas universidades públicas y privadas del Perú.

Es preciso aclarar, sin embargo, que la pregunta de investigación se focalizó en cómo las profesoras perciben y se representan las vivencias de las propias alumnas de ingeniería industrial; por tanto, no pretende indagar en torno a las experiencias que dichas docentes tuvieron en su propia formación como ingenieras años atrás, considerando que los contextos y tiempos son, evidentemente, muy distintos. Explorar estas percepciones podría ayudar a proponer estrategias $\mathrm{y}$ lineamientos generales y muy actuales conducentes a aumentar la participación de las mujeres jóvenes peruanas en esta importante subespecialidad de la ingeniería.

\section{1. ¿Qué dice la literatura sobre el género y las áreas STEM?}

Los investigadores se han preguntado a lo largo de varias generaciones en torno a las razones que explicarían la brecha de género en los campos STEM, y particularmente en las ingenierías. Esta pregunta cobra aún más interés en la medida que en la actualidad existe importante evidencia en torno al hecho que las mujeres suelen tener un rendimiento académico similar e incluso mejor que sus compañeros masculinos en las pruebas y actividades de estas áreas (Stoet y Geary, 2018). Sin embargo, también es sabido que van perdiendo interés conforme pasa el tiempo y la brecha de género se traslada a la educación superior y al trabajo, aunque se debe aceptar que son varios factores los que explicarían esta realidad (Reinking y Martin, 2018; Chávez et al., 2020).

En el caso peruano, por ejemplo, se menciona líneas arriba que apenas el 33\% de los graduados en ingeniería industrial en el año 2017 fueron mujeres. Por su parte, la tasa de pos graduación femenina (maestría y doctorado) en el año 2017 de todas las ingenierías fue de apenas $26,1 \%$ frente a la masculina (SUNEDU, 2019).

El panorama en otros países latinoamericanos no resulta diferente. En Argentina, por ejemplo, las estudiantes de ingeniería industrial rondaban el 25,4\% durante el 2016, el doble de lo que representaban hace diez años. En México y Brasil apenas 2 o 3 de cada 10 ingenieros son mujeres, mientras que en Uruguay y Costa Rica las mujeres representan el $32 \%$ y $27 \%$ de los ingenieros, respectivamente. La presencia femenina en la graduación de las ingenierías en Chile y Colombia no resulta más auspiciosa, puesto que cuentan con una representación también de alrededor del 30\% (Lerena y Páez, 2019). 
Con la finalidad de entender mejor esta problemática, se revisaron algunas tesis que podrían explicar esta realidad y que terminarían afectando la presencia femenina en los campos STEM, a fin de vislumbrar qué tareas son las más urgentes a fin de reducir la brecha de género en las ingenierías.

\subsection{Estereotipos de género y socialización}

$\mathrm{Si}$ bien no existe uniformidad de criterios en los investigadores en cuanto a las razones que podrían explicar la baja representación femenina en las áreas STEM, sí se pueden encontrar algunas ideas generales que intentan abordarla y entenderla. Reinking y Martin (2018), por ejemplo, proponen tres teorías generales al respecto: La socialización basada en estereotipos de género, los grupos de iguales, y los estereotipos propios de los profesionales STEM.

Otros estudios, a su vez, gravitan en torno al rol de la maternidad como un factor determinante para el éxito de las mujeres presentes en las áreas STEM y su continuidad profesional (Goulden, Mason y Frasch, 2011; Herman y Lewis, 2012), a los retos que éstas deben enfrentar para equilibrar su carrera profesional y vida familiar, así como a la necesidad de apoyo y acompañamiento requerido en tales circunstancias por parte de sus centros laborales (Howe-Walsh et al., 2016).

La socialización basada en estereotipos de género (Eccles, 2015) responde a las prácticas de socialización imperantes desde la niñez en muchos países occidentales. Estas prácticas se basan en ciertos estereotipos inculcados por padres y profesores desde pequeños y se sostienen en la idea del dominio masculino, así como la sumisión femenina. La familia, la escuela y los espacios de socialización, reproducen ciertos estereotipos de género que influyen en la orientación vocacional y la formación universitaria de las niñas, generando mayores niveles de desigualdad. Los procesos de socialización y de estímulo en hombres, así como en mujeres durante la infancia son importantes para el acercamiento a las ciencias. Tal acercamiento y desarrollo del interés científico son tan significativos como la metodología empleada en la enseñanza de las ciencias (Rodríguez, 2008).

Algunos de estos estereotipos, por ejemplo, refuerzan la idea que los niños son mejores en matemáticas (Gunderson et al., 2011) y por tanto se termina minusvalorando el interés de las niñas hacia áreas vinculadas, entre otras profesiones, a las ingenierías. Esta marginación reduce la legitimidad que una mujer puede tener cuando es formada en áreas STEM, así como su capacidad de liderazgo, de hacerse escuchar y de asumir una actitud más activa, tanto dentro del aula como en el mercado laboral (Regner et al., 2014). Entre otras consecuencias, la socialización basada en estereotipos de género, influiría desfavorablemente en la motivación y rendimiento obtenido por las niñas en las áreas STEM (Shapiro y Williams, 2012), y principalmente explicarían en parte el abandono femenino dentro de estas áreas (Dasgupta y Stout, 2014).

\subsection{Grupos de iguales y coetáneos}

Por otra parte, existe también la tesis en torno a que los llamados grupos de iguales o coetáneos podrían explicar la brecha de género en las ingenierías y áreas STEM (Reinking y Martin, 2018). Diversas investigaciones apuntan en torno a la fuerte influencia que los compañeros de los niños y adolescentes pueden generar en sus conductas y comportamientos sociales (Crosnoe et al., 2008; Van Hoorn et al., 2016). Esto se traduce, desde luego, en la motivación para la vida académica y su consecuente éxito dada la vulnerabilidad que los adolescentes presentan frente a la opinión de los demás (You, 2011). Esta realidad se puede extrapolar también al área de las ciencias y las matemáticas, y a cómo los grupos de chicas pueden influir entre ellas para el éxito o fracaso de llevar ciertos 
cursos e incursionar en áreas STEM como las ingenierías (Leaper, Farkas y Brown, 2012).

Hallazgos como los de Oliveros et al. (2016), demuestran que las alumnas de ingeniería de una universidad mexicana, por ejemplo, carecen de un modelo a seguir; por lo que, proponen la inclusión de tales modelos femeninos a fin de incrementar la motivación y matrícula de las mujeres en esta área del conocimiento. Lamentablemente, la influencia de los pares no deja de estar exenta muchas veces de matices vinculados a los estereotipos de la socialización anteriormente mencionados, puesto que los mismos grupos de adolescentes son portadores de aquellos imaginarios simbólicos que sus padres y profesores les terminan inculcando socialmente.

\subsection{Estereotipos profesionales}

Los estereotipos de género inculcados desde la niñez, se impregnan también en la vida adulta y más concretamente en su relación a profesiones como la ingeniería. Estos estereotipos se corresponden a los rasgos o características que los profesionales de estas áreas deberían tener. Cheryan, Master y Meltzoff (2015); así como Starr (2018), explican cómo la introversión y aislamiento social están fuertemente vinculados a los profesionales de la ingeniería, y cómo dichos rasgos de personalidad podrían generar aversión en las mujeres que suelen ser más sociables y abiertas. Además, resulta interesante cómo los medios de comunicación influyen en la construcción, reproducción y transmisión de estos estereotipos profesionales que no representan cualidades valoradas por el sexo femenino y que, por el contario, son un escollo asumido e históricamente arraigado contra los que deben luchar las mujeres que abrazan profesionalmente las áreas STEM (Steinke, 2017).

Paradójicamente, la ingeniería industrial estaría posicionada dentro de las demás subespecialidades como una de las menos "masculinas" (Blosser, 2017). Desde muy temprano se construyen imágenes de género para posicionar a algunas carreras como más o menos "masculinas". Si bien la ingeniería industrial demanda competencias técnicas y matemáticas, está asociada como más afín a las mujeres, sobre la base de creencias culturales sobre el género femenino: La capacidad de comunicar y de relacionarse interpersonalmente (Brawner et al., 2012; Blosser, 2017).

Las mujeres que optan por una profesión de corte masculino también se enfrentan a barreras que dificultan su desarrollo profesional. Por ejemplo, el sentirse sometidas a pequeñas y superficiales concesiones por parte del grupo masculino dominante (Martínez, 2018), o el ser víctimas de ciertas formas encubiertas de discriminación, como un clima laboral frío o ausencia de efectivas políticas de retención (Bannikova, Baliasov, y Kemmet, 2018). Esto provoca que afronten condiciones de excesiva visibilidad, atrayendo mayor atención sobre las actividades que realizan y por lo tanto una mayor presión sobre su desempeño.

En este proceso de aislamiento del grupo dominante, muchas veces terminan adaptándose, así como aceptando sus valores y finalmente los asimilan, siendo colocadas en una posición de subordinación; recordándoles el lugar que deben ocupar en esta profesión eminentemente masculina. Esta realidad podría terminar en la falta de confianza de las mujeres cuando incursionan en áreas STEM, puesto que la visión masculina prevalece socialmente en el mundo de la ingeniería (Olmedo et al., 2018); a pesar que en Perú se han diseñado e implementado políticas públicas "orientando los esfuerzos de las autoridades en generar oportunidades para alcanzar propuestas educativas, sin distinción de clases, ni de género" (Castillo-SantaMaría et al., 2020, p.84).

\subsection{La maternidad y el ejercicio profesional}

Finalmente, las barreras que encuentran las mujeres con niños y la maternidad en 
Ruiz-Ruiz, Marcos Fernando; Noriega-Aranibar, María Teresa y

Pease-Dreibelbis, María Angélica

Brecha de género en la graduación de ingenieras industriales peruanas

general, constituye una tesis menos explorada en torno a la baja representación femenina en las áreas STEM. La evidencia demuestra, entre otras cosas, que las ingenieras que son madres tienen menos oportunidades en el ejercicio de su carrera, así como al acceso a mentores y redes académicas y profesionales al interior de las organizaciones (Howe-Walsh et al., 2016). Las redes académicas cobran importancia en el desarrollo profesional y no tendrían que contraponerse a las obligaciones propias de la maternidad.

Muy por el contrario, las organizaciones deberían encontrar los mecanismos para favorecerlas a todo nivel: Tanto formales como informales (Barnard et al., 2010; Williams y Ceci, 2012; Llanes et al., 2020). Esta realidad, explicaría también las razones de por qué tan pocas mujeres alcanzan puestos de liderazgo en las ingenierías y cualquier otra área STEM (Nazemi, Mortazavi y Borjalilou, 2012; Nguyen, 2013; Ceci et al., 2014; Howe-Walsh y Turnbull, 2016).

El acompañamiento institucional de las mujeres a través de mentores, es un tema percibido de forma diferente por aquellas que son madres y las que no. Howe-Walsh et al. (2016), sugieren la importancia de contar con un adecuado acompañamiento al interior de la organización que dé apoyo a aquellas mujeres que tienen que aprender a equilibrar la maternidad con sus actividades profesionales.

\section{Metodología}

El enfoque metodológico empleado en el proceso de explorar las percepciones de las profesoras ingenieras industriales peruanas en torno a la baja representación femenina en este programa de pregrado, fue cualitativo. La investigación cualitativa, es útil cuando el problema de la investigación no ha sido abordado o escasamente explorado en el contexto seleccionado (Sofaer, 1999). Adicionalmente, este tipo de abordaje metodológico permite captar información como sentimientos, motivaciones o valores que, difícilmente, pueden ser detectados bajo una metodología cuantitativa (Berkwits y Inui, 1998).

En consecuencia, y dada la nula información en torno a las percepciones sobre la brecha de género en el campo de la ingeniería industrial peruana, se optó por esta aproximación cualitativa de naturaleza exploratoria y descriptiva del contexto de la investigación. El objetivo de este estudio no fue buscar la generalización per se (Soilemezi y Linceviciute, 2018); por el contrario, se buscó explorar y describir a fin de entender profundamente qué sistemas de significados alrededor del género gravitan de forma subrepticia en las percepciones de las ingenieras docentes en torno a sus propias alumnas y a la baja representación en el programa estudiado.

Para la investigación se efectuaron entrevistas semiestructuradas a 24 mujeres ingenieras industriales y docentes de pregrado de diferentes universidades peruanas. La selección de las entrevistadas obedeció a criterios vinculados a su titulación como ingenieras industriales, a su experiencia como docentes de alumnas de pregrado en programas de ingeniería industrial, y a la accesibilidad para efectuar las entrevistas; por lo cual, la escogencia de las participantes fue intencional y no probabilística. El grupo no estuvo restringido a profesoras procedentes de una ciudad específica, procurando una muestra lo más variada posible a fin de recabar distintas percepciones en torno al tópico investigado.

A pesar de ello, hubo dificultad en encontrar docentes con las características indicadas, dada la escasa presencia de docentes mujeres en los programas de ingeniería de algunas universidades y la ausencia de facultades de la especialidad en muchas regiones del país. Por tal razón, fueron entrevistadas docentes ingenieras principalmente de la ciudad de Lima, con algunas pocas representantes de las ciudades de Arequipa, Piura y Cuzco. Tampoco se discriminó si eran nombradas o contratadas a tiempo completo o parcial. Adicionalmente, el grupo seleccionado provenía de universidades tanto públicas como privadas, y de facultades 
de ingeniería industrial de importante trayectoria e influencia a nivel nacional.

Las profesoras fueron contactadas a través de medios electrónicos o telefónicos, con el fin de pactar una entrevista personal o telefónica, según cada caso, que sería grabada digitalmente bajo su consentimiento. Tanto para las entrevistas personales como telefónicas, se procuró coordinar un espacio $\mathrm{y}$ momento adecuados, un tiempo prudente y, de ser el caso, un teléfono privado a fin de garantizar la confiabilidad y calidad de las entrevistas. En algunos casos se utilizó las referencias de ellas mismas para contactar con otras posibles participantes (bola de nieve). La Tabla 1, presenta la relación de las 24 docentes ingenieras industriales entrevistadas con algunas características en torno a sus perfiles y procedencia. Los nombres de las mismas, así como sus universidades de origen, han sido mantenidos en el anonimato por razones de confidencialidad y a fin de permitirles un espacio de diálogo más sincero y abierto.

\section{Tabla 1}

Participantes (perfiles y características generales)

\begin{tabular}{|c|c|c|c|c|c|c|}
\hline Código ${ }^{*}$ & Edad (años) & $\begin{array}{c}\text { Tiempo de } \\
\text { servicio (años) }\end{array}$ & Maternidad & Tipo de universidad & Dedicación laboral & Ciudad \\
\hline $\mathrm{N}^{\circ} 1$ & 55 & 20 & SI & Pública & T. Completo & Lima \\
\hline $\mathrm{N}^{\circ} 2$ & 57 & 36 & SI & Pública & T. Completo & Lima \\
\hline $\mathrm{N}^{\circ} 3$ & 58 & 42 & SI & Pública & T. Completo & Lima \\
\hline $\mathrm{N}^{\circ} 4$ & 65 & 33 & SI & Pública & T. Completo & Lima \\
\hline $\mathrm{N}^{\circ} 5$ & 55 & 20 & SI & Pública & T. Completo & Arequipa \\
\hline$N^{\circ} 6$ & 55 & 21 & SI & Pública & T. Completo & Piura \\
\hline $\mathrm{N}^{0} 7$ & 50 & 27 & NO & Pública & T. Completo & Lima \\
\hline $\mathrm{N}^{\circ} 8$ & 52 & 26 & SI & Privada & T. Completo & Piura \\
\hline $\mathrm{N}^{\circ} 9$ & 45 & 23 & SI & Privada & T. Completo & Cuzco \\
\hline $\mathrm{N}^{\circ} 10$ & 47 & 19 & SI & Privada & T. Completo & Piura \\
\hline $\mathrm{N}^{\circ} 11$ & 53 & 19 & SI & Privada & T. Completo & Piura \\
\hline $\mathrm{N}^{\circ} 12$ & 26 & 2 & NO & Privada & T. Parcial & Lima \\
\hline $\mathrm{N}^{\circ} 13$ & 56 & 24 & NO & Privada & T. Completo & Lima \\
\hline $\mathrm{N}^{\circ} 14$ & 49 & 29 & SI & Privada & T. Parcial & Lima \\
\hline $\mathrm{N}^{\circ} 15$ & 45 & 5 & SI & Privada & T. Completo & Lima \\
\hline $\mathrm{N}^{\circ} 16$ & 45 & 8 & SI & Privada & T. Completo & Lima \\
\hline $\mathrm{N}^{\circ} 17$ & 47 & 11 & SI & Privada & T. Parcial & Piura \\
\hline $\mathrm{N}^{\circ} 18$ & 45 & 20 & NO & Privada & T. Parcial & Lima \\
\hline $\mathrm{N}^{\circ} 19$ & 32 & 4 & NO & Privada & T. Completo & Lima \\
\hline $\mathrm{N}^{\circ} 20$ & 44 & 4 & NO & Privada & T. Completo & Lima \\
\hline $\mathrm{N}^{\circ} 21$ & 38 & 2 & SI & Privada & T. Parcial & Arequipa \\
\hline $\mathrm{N}^{\circ} 22$ & 28 & 5 & NO & Privada & T. Parcial & Lima \\
\hline $\mathrm{N}^{\circ} 23$ & 35 & 3 & SI & Privada & T. Completo & Arequipa \\
\hline $\mathrm{N}^{\circ} 24$ & 44 & 4 & SI & Privada & T. Completo & Arequipa \\
\hline
\end{tabular}

Nota: *Todas las participantes fueron profesoras en ejercicio en el programa de ingeniería industrial y con grado y/o posgrado en dicha área.

Fuente: Elaboración propia, 2021.

La nacionalidad de las entrevistadas no constituyó un factor relevante, dado que todas ellas son nacidas en el Perú y/o llevan muchos años ejerciendo la actividad docente en este contexto. Sin embargo, la presencia de algunas docentes de ciudades distintas a Lima tuvo el fin de enriquecer las perspectivas y miradas desde distintos ángulos y contextos de país. Considerar este aspecto es importante puesto que la nacionalidad y cultura de procedencia de una entrevistada podría influenciar en sus percepciones respecto al enfoque de género dentro de la ingeniería que se quiere investigar (Downey y Lucena, 2005).

La selección de la técnica de la entrevista obedeció a su utilidad como una de las principales fuentes primarias para la recolección de datos en los estudios cualitativos 
(Creswell, 2007; Yin, 2009; Merriam y Tisdell, 2015). Éstas fueron realizadas entre los meses de mayo y diciembre del 2019. Asimismo, la guía de entrevista fue elaborada previamente tomando como base cinco dimensiones vinculadas a la pregunta de investigación: Características de las docentes entrevistadas, condiciones personales y de género de sus alumnas, el contexto académico de sus estudiantes, su contexto laboral y la brecha de género en la graduación de ingenieros industriales en el Perú.

A cada una de las dimensiones anteriormente descritas se le asignó entre dos a tres preguntas más específicas que permitieron explorarlas a mayor detalle. Durante las entrevistas, sin embargo, se pudo profundizar con libertad sobre algunas de las preguntas realizadas con la intención de ahondar en las motivaciones e ideas subyacentes.

La guía de entrevista, en cuanto a dimensiones y contenidos, fue elaborada exclusivamente para esta investigación y validada a través de pruebas piloto con ayuda de dos profesoras investigadoras e ingenieras industriales que no participaron posteriormente de la investigación. Dichas pruebas piloto, permitieron recabar información valiosa orientada a la optimización del instrumento, a su focalización y a su mejor comprensión para las participantes. La Tabla 2, presenta de forma resumida las cinco dimensiones de la entrevista con sus objetivos y algunas preguntas tipo.

Tabla 2

Dimensiones de análisis

\begin{tabular}{|c|c|c|c|}
\hline & Dimensión & Objetivos & Preguntas tipo \\
\hline 1. & $\begin{array}{l}\text { Características de las } \\
\text { docentes entrevistadas. }\end{array}$ & $\begin{array}{l}\text { Conocer aspectos generales de las } \\
\text { profesoras entrevistadas. Entre ellos, } \\
\text { el tiempo que llevan enseñando en el } \\
\text { programa, sus áreas de trabajo y la } \\
\text { percepción general que tienen en torno a } \\
\text { la conformación de sus aulas desde una } \\
\text { perspectiva de género. }\end{array}$ & $\begin{array}{l}\text { ¿Cuánto tiempo lleva enseñando en la } \\
\text { especialidad de ingeniería industrial, } \\
\text { en qué ciclos y qué áreas? ¿Ha notado } \\
\text { algún cambio en la proporción de } \\
\text { hombres y mujeres estudiantes de dicha } \\
\text { especialidad en los últimos años? }\end{array}$ \\
\hline 2. & $\begin{array}{l}\text { Condiciones } \\
\text { personales y de género } \\
\text { de las alumnas del } \\
\text { programa. }\end{array}$ & $\begin{array}{l}\text { Analizar la percepción de las entrevistadas } \\
\text { en torno a las razones para el mayor o } \\
\text { menor grado de dificultad que enfrentan } \\
\text { sus alumnas estudiantes del programa de } \\
\text { ingeniería industrial por su condición de } \\
\text { género. }\end{array}$ & $\begin{array}{l}\text { ¿Considera que, para una alumna suya, } \\
\text { por su condición de mujer, es más fácil, } \\
\text { más difícil o exactamente igual que para } \\
\text { un hombre estudiar una carrera como } \\
\text { ingeniería industrial en el Perú? ¿Por } \\
\text { qué? }\end{array}$ \\
\hline 3. & $\begin{array}{l}\text { Contexto académico } \\
\text { de las alumnas del } \\
\text { programa. }\end{array}$ & $\begin{array}{l}\text { Examinar la existencia o no de diferencias } \\
\text { en cuanto a los desempeños académicos } \\
\text { en función al género y conocer de } \\
\text { experiencias relacionadas a exclusión o } \\
\text { discriminación en dicho contexto. }\end{array}$ & $\begin{array}{l}\text { ¿Considera que hay diferencias en } \\
\text { cuanto a desempeño académico entre } \\
\text { estudiantes de ingeniería industrial por } \\
\text { razones de género? De ser el caso, ¿a qué } \\
\text { factores los atribuye? }\end{array}$ \\
\hline 4. & $\begin{array}{l}\text { Contexto laboral de las } \\
\text { alumnas del programa. }\end{array}$ & $\begin{array}{l}\text { Analizar las percepciones en torno al } \\
\text { contexto laboral que enfrentan o que } \\
\text {-se intuye- enfrentarán las alumnas } \\
\text { del programa. Además conocer de } \\
\text { experiencias referenciadas sobre } \\
\text { discriminación por razones de género en } \\
\text { las prácticas laborales de las estudiantes. }\end{array}$ & $\begin{array}{l}\text { ¿Considera que les espera alguna } \\
\text { situación laboral particular a sus alumnas } \\
\text { de ingeniería industrial, por su condición } \\
\text { de mujeres? De ser el caso, ¿cuál(es) y a } \\
\text { qué factores la(s) atribuye? }\end{array}$ \\
\hline 5. & $\begin{array}{l}\text { Brecha de género } \\
\text { en la graduación de } \\
\text { ingenieros industriales } \\
\text { en el Perú. }\end{array}$ & $\begin{array}{l}\text { Explorar, en base a las experiencias de } \\
\text { las entrevistadas y luego de examinar } \\
\text { información estadística, las razones que } \\
\text { podrían explicar la brecha de género en la } \\
\text { graduación del programa y las estrategias } \\
\text { convenientes para reducirla. }\end{array}$ & $\begin{array}{l}\text { Según su experiencia como formadora ¿a } \\
\text { qué se podría atribuir la desigualdad en } \\
\text { la postulación de mujeres a las carreras } \\
\text { de ingeniería industrial en el Perú? } \\
\text { Mencione tres mecanismos que usaría } \\
\text { para alentar a las futuras generaciones de } \\
\text { mujeres a estudiaringeniería industrial. }\end{array}$ \\
\hline
\end{tabular}

Fuente: Elaboración propia, 2021. 
Todas las entrevistas fueron pactadas con unos días de anticipación y tomaron un tiempo variable de entre 15 y 35 minutos. Se realizaron de forma presencial, a excepción de las concertadas con profesoras de universidades muy distantes de Lima; a éstas, por razones de accesibilidad, se les tuvo que entrevistar telefónicamente.

Para un mejor tratamiento de la información, todas las respuestas fueron grabadas de forma digital a fin de ser escuchadas posteriormente con el objeto de analizar, dentro de cada dimensión y de la forma más fidedigna, las ideas subyacentes, así como las convergencias y divergencias en las opiniones sobre el tema de la investigación. La grabación de las entrevistas no solo posibilitó la transcripción de las opiniones vertidas sobre algún tema de la investigación, sino la descripción posterior de los hallazgos y los contextos de la propia investigación con más exactitud (Aguirre y Jaramillo, 2015).

Asimismo, para el análisis de los datos recabados y por la naturaleza social del fenómeno estudiado, se escogió una aproximación metodológica constructivista e influenciada por la teoría fundamentada. Esta aproximación teórica, permitió identificar y codificar categorías derivadas de los datos a partir de un método comparativo constante entre diversos episodios de las entrevistas bajo un proceso eminentemente inductivo, para luego agruparlas en temas y patrones (Glaser y Strauss, 2017; Hammersley, 2018). Finalmente, se procedió a consolidar los hallazgos al interior de cada una de las dimensiones de análisis previamente definidas, para luego elaborar el reporte de resultados y su correspondiente análisis.

Algunos temas no previstos desde el inicio, y que surgieron de manera emergente, fueron también considerados. Este procedimiento es propio de este tipo de abordaje cualitativo, puesto que permite una mayor aproximación a las percepciones reales, así como aprender de las experiencias y perspectivas personales de los entrevistados (DiCicco-Bloom y Crabtree, 2006).

\section{Resultados}

Las entrevistadas perciben aún como incipiente la presencia de mujeres en las facultades de ingeniería industrial peruanas; casi todas estiman la proporción femenina entre un $30 \%$ y $40 \%$. Esta percepción coincide con los datos estadísticos de la educación profesional en el país (SUNEDU, 2019). Difícilmente se atreven a asegurar una paridad de género en el aula.

Sin embargo, reconocen que la presencia femenina en la especialidad se ha ido incrementado si es que se compara con la de diez o veinte años atrás, cuando ellas mismas eran estudiantes; hecho que es avalado por las estadísticas oficiales. A pesar de esos avances, consideran que aún hay un arduo trabajo por hacer en el país a fin de seguir cerrando la brecha de género en las áreas STEM y más específicamente, en ingeniería industrial.

\subsection{Género y facilidades para el acceso a estudios de ingeniería industrial en el Perú}

Las entrevistadas refieren que, si bien no existen limitaciones formalmente establecidas en cuanto al acceso de las mujeres para seguir una carrera de ingeniería industrial en el Perú, éstas pueden encontrar mayores dificultades que sus pares varones a lo largo de sus estudios profesionales. Aquellas entrevistadas que coincidieron en este aspecto, relacionaron directamente el grado de dificultad de seguir estudios en las áreas STEM, que se reconocen como exigentes y demandantes, con las obligaciones familiares y la responsabilidad de ser madres, según sea el caso. Por su parte, esta realidad explicaría también por qué muchas jóvenes optan por carreras que la sociedad reconoce como más llevaderas o menos exigentes que las de las áreas STEM.

$\mathrm{Al}$ respecto, algunas de las entrevistadas manifiestan: "He sido testigo, en muchos casos, de alumnas que han salido embarazadas 
y eso las ha obligado a dilatar sus estudios" (Entrevistada No.16, Lima, 45 años, U. Privada); “(...) las mujeres, a veces, tienen que ayudar en casa, porque en el Perú aún tenemos la idea de que los hombres deben tener más privilegios" (Entrevistada No.23, Arequipa, 35 años, U. Privada); "A la mujer la sociedad la educa a estudiar siempre algo más fácil, más light. Eso porque va a tener que dedicarse a los hijos. Así somos educadas" (Entrevistada No.15, Lima, 45 años, U. Privada).

Existe, además, una percepción en las docentes que diferencia a las estudiantes de ingeniería industrial de universidades privadas y públicas en el Perú. En este sentido, perciben que las jóvenes que provienen de hogares con mayores ingresos económicos, y por tanto pueden acceder a la educación de una universidad privada, cuentan normalmente con un mayor apoyo familiar para seguir los estudios en áreas STEM como la ingeniería industrial. No es de extrañar entonces que las docentes observan siempre una proporción femenina mayor en estos contextos educativos privados que en los de las universidades públicas. Asimismo, dicho apoyo familiar concuerda con una actitud más segura y participativa, aunque siempre en menor medida que la de los varones.

A pesar de ello, las chicas suelen muchas veces preguntar o aportar menos en clase y en espacios públicos, percibiéndose más tímidas. En ocasiones, incluso, los mismos docentes refuerzan muchas veces este escenario a través de sus propias prácticas pedagógicas.

En las universidades particulares no hay diferencias significativas [entre hombres y mujeres estudiantes] o no se perciben por el empoderamiento que viene del hogar. En cambio, en niveles socioeconómicos con menor formación sí se hace evidente una diferencia. Esa diferencia se ve mucho en las universidades nacionales [públicas]. (Entrevistada No.10, Piura, 47 años, U. Privada)

Cuando es un espacio público, a diferencia de uno privado como las asesorías, los varones siempre aportan más. Las chicas a veces no preguntan, se sienten muchas veces 'disminuidas' [retraídas] frente a los varones. Los profesores [hombres] 'disminuyen' [retraen] un poco a las chicas. A mí me ha pasado. El trato [a los estudiantes varones] es diferente, especialmente si son docentes mayores. (Entrevistada No.14, Lima, 49 años, U. Privada)

Otra de las entrevistadas sostuvo: "Si bien no he sido testigo de discriminación, sí es evidente que los profesores [hombres] son más receptivos con los [alumnos] varones que con las mujeres" (Entrevistada No.12, Lima, 26 años, U. Privada).

\subsection{Percepciones sobre el contexto de desarrollo académico}

Resulta casi unánime la percepción de las docentes ingenieras en torno al hecho que no hay diferencias significativas en cuanto a las capacidades de hombres y mujeres estudiantes de ingeniería industrial. En todo caso, aquellas que perciben algún tipo de diferencia en cuanto al rendimiento académico, la correlacionan, por un lado, con la timidez o falta de confianza que algunas chicas pudieran tener y que debe ser oportunamente atendida; y por otro, por el contexto socioeconómico de la escuela y la familia de procedencia. Esta última refuerza, en algunas circunstancias, ciertos roles de género para las jóvenes estudiantes que están más vinculados a las obligaciones familiares como el deber de ser buenas madres y esposasantes que el hecho de desarrollarse como profesionales.

En ese sentido, manifiestan que: “Académica e intelectualmente las capacidades son exactamente las mismas. He tenido grupos donde las mujeres han tenido mejores calificaciones y otros en los que viceversa" (Entrevistada No.15, Lima, 45 años, U. Privada). "A muchas chicas procedentes de colegios religiosos las forman más en valores que en contenidos. Siento que las forman para casarse, y vienen para buscar esposo. Porque así las forman todavía" (Entrevistada No.14, Lima, 49 años, U. Privada).

Por otra parte, y a pesar de no percibirse diferencias en cuanto al rendimiento académico 
por razones estrictamente naturales a lo largo de los estudios en el área de interés, si existió un patrón casi unánime entre las entrevistadas en cuanto a la percepción respecto a la actitud y desempeño que muestran las chicas a lo largo de sus estudios. Esto lo atribuyen, más que a una cualidad de naturaleza biológica, a una exigencia a la que el entorno 'machista' de la sociedad peruana las obliga. Así, aquellas mujeres que escogen una carrera como la ingeniería industrial en un contexto social como el peruano saben que tienen que lidiar y competir continuamente en un espacio eminentemente masculino.

Esta realidad las empodera y se constituye como el catalizador que las hace ser percibidas por sus profesoras como más ordenadas, cumplidas, responsables, organizadas y disciplinadas. En muchos casos, incluso, como extremadamente competitivas y 'mandonas' o déspotas.

Las mujeres suelen ser más dedicadas en su forma de estudio, más cuidadosas, más pulcras. Se preparan más para una exposición, por ejemplo, y son más prolijas. Cuando en un grupo hay una mujer, ellas imponen orden y son más productivas. (Entrevistada No.13, Lima, 56 años, U. Privada)

Las mujeres son muy competitivas. Tienen que esforzarse más por el machismo peruano, y son capaces de hacerlo bien. Suelen ser detallistas, responsables. Una mujer muy dócil, difícilmente estudia esta carrera en el Perú. Las mujeres saben que tienen que competir más. Los hombres no tienen que demostrar nada. (Entrevistada No.16, Lima, 45 años, U. Privada)

En contraposición a lo anteriormente señalado, existe la percepción enraizada respecto al carácter más relajado y distendido de los estudiantes varones de ingeniería industrial en comparación a las mujeres. Una realidad, especialmente percibida por las docentes de universidades públicas antes que privadas, se centra en el carácter de irresponsabilidad asociado a muchos estudiantes varones. Esta irresponsabilidad se arraiga en ciertos patrones culturales y sociales vinculados al consumo de bebidas alcohólicas y a los juegos de apuestas. Esto está más arraigado en algunos contextos alejados de la ciudad capital. "Algunos estudiantes [varones] descuidan sus clases porque se van a jugar, a tomar o realizan apuestas. Las estudiantes mujeres salen menos. Tienen menos vicios que los alumnos varones, son más dedicadas. Por eso las califican como 'sobonas' [adulonas]" (Entrevistada No.7, Lima, 50 años, U. Pública).

Finalmente, se detecta una sutil percepción en cuanto a un temor e inseguridad frente a los números y las matemáticas, que termina siendo más marcada en las jóvenes estudiantes que en sus pares varones. Esto sin embargo se atribuye, antes que, a una realidad de tipo natural, al estereotipo de creer que las áreas STEM son un terreno exclusivamente masculino $\mathrm{y}$, por tanto, los hombres son mejores en números. A esto se añade también, las deficiencias en la formación escolar con las que muchas veces llegan los jóvenes a la universidad, especialmente cuando provienen de colegios exclusivamente de mujeres.

A veces, desde la familia, viene la creencia de que la ingeniería es solo para los varones. Eso genera miedo a las matemáticas en las jóvenes. Pero sin duda, hay que revisar también cómo se está enseñando en las escuelas. (Entrevistada No.4, Lima, 65 años, U. Pública)

Otra de las participantes menciono: "Creo que las matemáticas asustan a veces a las mujeres y no las preparan bien desde el colegio. A muchas chicas procedentes de colegios religiosos las forman más en valores que en contenidos" (Entrevistada No.14, Lima, 49 años, U. Privada).

\subsection{Contexto laboral para las industriales peruanas desde un enfoque de género}

La percepción en cuanto a las condiciones laborales que les espera a las futuras ingenieras industriales en el Perú, es quizás la que termina siendo más crítica $\mathrm{y}$, en algunos casos, un tanto hostil y menos auspiciosa. La primera idea general que se infiere de las opiniones de las entrevistadas, 
es que existe un marcado privilegio de lo masculino en el contexto laboral peruano, al menos en el caso de la ingeniería industrial. Tal privilegio tiene varias aristas a saber: Desde el tipo de trabajo al que se puede acceder como mujer, pasando por las injustas diferencias salariales frente a sus pares masculinos, y la maternidad, vista como un estigma que las empresas que contratan mujeres ingenieras industriales deben evitar; hasta algunos curiosos imaginarios supersticiosos en torno a la femineidad y el ejercicio profesional de la ingeniería.

En cuanto al tipo de trabajo, se infiere de las entrevistas con absoluta unanimidad, que las áreas de planta industrial, producción y técnicas están casi vetadas para las mujeres industriales. La justificación principal, enraizada en el imaginario empresarial e industrial peruano, radica en el hecho que una mujer debe primero demostrar su capacidad para un trabajo de esa naturaleza. Asimismo, debe demostrar su autoridad y que puede hacerse respetar frente a los operarios y obreros de planta sin ser temerosa o pusilánime.

Las mujeres somos brillantes, pero a veces terminamos en áreas más de oficina. En las empresas hay muchos prejuicios y nos vetan la posibilidad de ascender a posiciones donde, por ejemplo, tenemos que viajar, trabajar con obreros o en planta industrial. (Entrevistada No.17, Piura, 47 años, U. Privada)

En producción hay discriminación. Piensan que una mujer es incapaz de manejar una máquina. Nuestro país es aún 'machista' y muchos no están preparados para recibir órdenes de una mujer. A los hombres muchas veces les cuesta reconocer que una mujer está jerárquicamente por encima de ellos. (Entrevistada No.18, Lima, 45 años, U. Privada)

Las áreas empresariales restringidas al trabajo femenino de una ingeniera industrial son más las administrativas y las de gestión. Ese estereotipo de la 'mayor prolijidad' las restringe muchas veces de forma exclusiva a ciertas áreas de la ingeniería y las excluye de otras tantas. Por tal razón, es más común ver mujeres ingenieras industriales peruanas desempeñándose en áreas de servicios como la banca, los recursos humanos, servicios comerciales, retails y logística; donde tienen mayor aceptación según las experiencias de las entrevistadas.

No sucede lo mismo en áreas vinculadas a la manufactura, por ejemplo. Hay una fuerte coincidencia en las entrevistadas en cuanto al hecho que se debe convivir con un 'machismo' permanente y muy enraizado en la organización del contexto laboral peruano. Esta realidad la evidencian con mayor intensidad las docentes que trabajan con alumnas de universidades públicas: "En las primeras prácticas [mis alumnas] han sentido que los trabajos más importantes o relevantes se los daban a los varones. Ellas, por ser mujeres, estaban confinadas a las oficinas para ordenar y archivar papeles" (Entrevistada No.10, Piura, 47 años, U. Privada).

El machismo es permanente en el mundo laboral peruano. Hay más varones que mujeres y ellas ocupan trabajos administrativos. Una mujer encuentra más oportunidades en gestión o recursos humanos. En general, áreas de la ingeniería que no implican producción ni manufactura (Entrevistada $\mathrm{N}^{\circ} 21$, Arequipa, 38 años, U. Privada)

El tema salarial también está fuertemente influenciado por los privilegios masculinos del entorno laboral peruano para las futuras ingenieras industriales. Algunas de las entrevistadas tienen la sensación que las jóvenes industriales no perciben los mismos ingresos que sus pares masculinos precisamente porque se les niega la oportunidad de acceder a puestos más altos. Esto sucede, al parecer, en algunos contextos industriales y empresariales por las razones ya esgrimidas. "A la mujer le cuesta llegar a ocupar puestos altos. En algunos sectores se ofrece a los varones un mejor sueldo que a las mujeres. Los trabajos más importantes se los otorgan a los varones" (Entrevistada No.8, Piura, 52 años, U. Privada).

En cuanto a la relación entre el contexto laboral de las futuras ingenieras industriales y la maternidad, resulta convergente la percepción de varias de las entrevistadas en 
torno al carácter casi estigmatizado con el que lo presentan. Así, muchas empresas toman muy en cuenta esta realidad al momento de seleccionar a un postulante para alguna área de la empresa. Las licencias por maternidad y los permisos asociados al propio rol de ser madre, constituyen una incomodidad para muchas empresas y uno de los principales estigmas a los que se tienen que enfrentar las jóvenes egresadas de ingeniería para acceder a un puesto laboral en la empresa o el sector industrial.

Esto, desde luego, se articula a las limitaciones que les dan para acceder a puestos de mayor responsabilidad que podrían implicar muchas horas laborales, viajes y desde luego, mejores salarios. "Una vez me dijeron en un trabajo que no contrataban mujeres porque se embarazaban y piden permiso constantemente porque sus hijos se enferman" (Entrevistada No.14, Lima, 49 años, U. Privada).

La desigualdad laboral también se da porque la mujer es fértil, va a tener una familia y tendrá que dedicarse a los hijos en algún momento. Eso es así, porque así somos educados. Tuve una experiencia en la que, para contratarme, me pidieron una prueba de descarte de embarazo. (Entrevistada No.15, Lima, 45 años, U. Privada)

Finalmente, se advirtieron algunos comentarios que guardan relación con ciertos imaginarios simbólicos supersticiosos, la femineidad y el contexto laboral al que se tienen que enfrentar las futuras ingenieras. Este imaginario, enraizado especialmente en contextos alejados de la ciudad, presenta a la mujer ingeniera como portadora de "mala suerte" al ingresar a ciertos espacios laborales. Uno de ellos, y que atañe directamente a las futuras ingenieras industriales, se concentra en el trabajo propio de la industria minera.

Esto sin lugar a dudas también puede traer desventajas a aquellas jóvenes industriales interesadas en dicho sector: "No sé si esto puede influir, pero dicen que las mujeres [ingenieras] traen muy mala suerte si trabajan en la industria minera del país. Sin duda alguna hay muchos mitos que aún tenemos que desterrar" (Entrevistada No.20,
Lima, 44 años, U. Privada). Otra comenta: "Existe la idea de que las mujeres suelen ser más delicadas y, por ejemplo, en una mina, dan mala suerte. Es un mito" (Entrevistada No.19, Lima, 32 años, U. Privada).

\section{Discusión}

Los resultados confirman algunos hallazgos ya planteados en la literatura al respecto y, al mismo tiempo, propone algunos otros que emergen en la realidad. Pese a que las docentes tienen una percepción adecuada y coincidente con las estadísticas de cuánto participan las estudiantes en la carrera de ingeniería, consideran que esta presencia se está incrementando.

Al igual que lo planteado por Ames (2006); Rodríguez (2008); y Regner et al. (2014), las entrevistadas plantean que las mujeres tienden a exhibir actitudes y comportamientos en el devenir de las clases que las distinguen según género. Sin embargo, intersectan dichas diferencias con el hecho que sean estudiantes de universidades públicas o privadas.

De igual manera, al igual que en la literatura previa (Rodríguez, 2008; Guerrero y Rojas, 2017), las docentes identifican que las estudiantes tienen mayores presiones familiares que los varones. Sin embargo, éstas no obedecen únicamente a motivos vinculados a la conformación de sus propias familias por convivencia, matrimonio o maternidad, como en los trabajos de Goulden et al. (2011); y Herman y Lewis (2012), sino que las presiones provienen también de sus familias de origen. Dichas coacciones, sin embargo, afectan de manera distinta en función del tipo de universidad. Las mujeres de universidades públicas, tendrían una sobrecarga mayor de responsabilidades en casa que las llevan a tener más presiones que compiten con los estudios.

Dichos hallazgos remiten a los estudios de Portocarrero (1998), respecto a la existencia de mandatos generacionales diferenciados entre padres de sectores populares y de clases medias en el país. Así, mientras los 
padres y madres de adolescentes tardíos de clases medias. que se encuentran transitando a estudios post secundarios les darían el mandato que exploren, aprendan quiénes son, identifiquen una vocación y la alcancen; los de sectores populares, con mayores presiones y cargas económicas, darían mandatos que restringen dicha exploración y que más bien los lleva a pedirles que culminen sus estudios lo antes posible, mientras colaboran en sacar a la familia adelante.

En la misma línea, el estudio de Urbano (2018), que contrasta adolescentes de escuelas públicas y privadas, muestra que los primeros, incluyen entre sus responsabilidades futuras la idea de "sacar a la familia adelante"; hallazgo que ya había sido identificado en un estudio realizado una década antes (Pease, 1999). Lo interesante es que, en este caso, las docentes intersectan este consistente hallazgo de la literatura con diferencias de género, estableciendo que esta situación, que ya resulta problemático para las y los adolescentes de sectores populares, resultaría peor para las mujeres.

Como plantean Reinking y Martin (2018), las docentes identifican que los estereotipos de género y la educación recibida, son un importante condicionante de la presencia de las mujeres en la carrera de ingeniería industrial. Sin embargo, aparecen precisiones interesantes que dan cuenta de contextos de discriminación probablemente más explícitos que aquellos a los que refieren otros trabajos. Un factor recurrentemente identificado por las participantes, es el rol de los docentes universitarios, quienes, a través de sus expectativas, comentarios y prácticas pedagógicas, refuerzan el estereotipo que los varones son más competentes que las mujeres.

Los docentes "disminuyen" a las mujeres, tal como refiere una participante, de manera explícita. Ello parecería estar bastante naturalizado y poco cuestionado en el aula según reportan las entrevistadas. Al mismo tiempo, la aceptación de dichos discursos, el que no sea confrontado ni por las docentes ni por las estudiantes, mantendría todo en una suerte de calma que previene colocarlos en cuestión. De este modo, se naturalizarían dichas diferencias respecto a la competencia, afectando el sentido de autoeficacia de las estudiantes (Bandura, 1986). Ello resulta un hallazgo sumamente valioso si se atiende además que, en el Perú, ha habido una fuerte ofensiva de sectores ultra conservadores y anti derechos, respecto al enfoque de género en la educación a nivel básico (primario y secundario).

De hecho, se ha llegado a enjuiciar al Estado peruano para remover parte del currículo de educación sexual. Ello, sin embargo, no ha encontrado eco ni en los padres y madres de familia (Motta et al., 2017), ni en los estudiantes que demandan una educación sexual integral (Pease et al., 2019). Por su parte, dicha discusión pareciera no haber llegado a las aulas de la educación superior. Ni siquiera cuando, en los últimos años, han aparecido una serie de denuncias de violencia sexual hacia alumnas (ya sea contra docentes o estudiantes varones) en prestigiosas universidades de Lima.

Ahora bien, las propias participantes no se encuentran al margen de este contexto. Resulta muy revelador que cuando ellas refieren a las características de sus estudiantes mujeres y al por qué les cuesta desenvolverse en ciertos puestos de trabajo, señalan ciertas características estereotípicamente asociadas a las mujeres; como aquella delicadeza que no les permite empoderarse, o -por el contrariose refieren a ellas como competitivas y mandonas. Dichas características son, sin embargo, asociadas a la femineidad, no vistas como características personales distintivas de una u otra persona. Se estaría pues en medio de un proceso en el que las mismas docentes no han logrado desnaturalizar atributos asociados a los sexos (Ruiz, 1997).

Finalmente, las docentes sí aparecen mucho más críticas respecto a la adjudicación de menor competencia a las estudiantes respecto a sus pares varones. Ello tiene total sentido, en tanto sus propias vidas y experiencias dan cuenta que se puede ser competente en este campo. Identifican que opera en ellas, de manera muy marcada, estereotipos asociados 
a profesiones STEM, que suelen colocar en valor atributos típicamente asociados a la masculinidad (Reinking y Martin, 2018). Se muestran críticas ante este punto de vista y consideran que opera de manera bastante desfavorable en el sentido de autoeficacia de las estudiantes (Bandura, 1986).

Las reflexiones en este punto de las docentes, suelen centrarse en aquellas capacidades y características que las estudiantes tienen que afirmar o que se suele no esperar de ellas. Resulta curioso que no haya una reflexión respecto a la existencia de barreras de acceso, creadas por un sistema de inequidad donde la primacía masculina genera las expectativas y refuerza aquello coincidente con ellas. Es como si se discutiera la discriminación sin atender que hay una estructura patriarcal desigual en su origen que favorece a los varones sobre las mujeres, y por ello se refieren principalmente a cualidades o atributos de las mujeres que logran alcanzar éxito.

Ello sería problemático, en tanto, al no hacerse visible un contexto mayor que opera sobre las opciones que las mujeres en las ingenierías logran alcanzar, parecería que sus logros pudieran depender únicamente de su agencia, de su esfuerzo, de sus atributos y capacidades, y no de condiciones que las estimulan o inhiben.

Visibilizar dichas mediaciones y condicionantes, acompañar a las docentes a desnaturalizar ciertos atributos asociados a los géneros, así como trabajar de manera más explícita en la educación superior con un enfoque de género, que frene los tratos desiguales hacia las mujeres por parte de los docentes; serán tareas clave en términos de intervención para poder conseguir una mejor participación femenina en las ciencias e ingenierías.

\section{Conclusiones}

El presente estudio tuvo por finalidad analizar los motivos de la baja presencia de estudiantes mujeres en la ingeniería industrial peruana, ello a partir de las percepciones de las docentes de ingeniería. Las entrevistadas advierten la existencia de imaginarios concretos hacia las estudiantes de ingeniería que no se presentan con los estudiantes varones, entre ellos, las altas expectativas de desempeño por parte de su entorno familiar y académico, así como la atribución de características personales asociadas a su género que no necesariamente son atribuibles a todos los varones. Esta última realidad genera, incluso, un trato diferenciado por parte de los mismos docentes que tienden a disminuirlas en ciertos contextos.

A pesar que las participantes toman conciencia crítica de las subjetividades anteriormente descritas, evidencian una sobrevaloración general de las capacidades y competencias de sus estudiantes mujeres, así como del desarrollo de atributos que las lleva normalmente a conquistar el mercado laboral. Paradójicamente, dicha sobrevaloración gravita en las entrevistadas con mayor preponderancia que la crítica orientada a la toma de acciones, que eliminen barreras que excluyen a las mujeres de ciertos entornos de dominio masculino, como lo son las áreas STEM, en busca de una mayor equidad.

Finalmente, una limitación de este estudio radica en que solo aborda las percepciones y representaciones que las docentes ingenieras guardan respecto de sus propias estudiantes y no así desde otras miradas como las de las mismas alumnas. No obstante, esto abre algunas posibles líneas de indagación a partir de los resultados y reflexiones aquí presentadas. Por un lado, una investigación ampliada que se intersecte con las percepciones de las estudiantes de ingeniería; y por otro, un trabajo prospectivo de estrategias y lineamientos generales conducentes a incrementar la presencia de mujeres jóvenes en las áreas STEM y en la ingeniería industrial peruana.

\section{Referencias bibliográficas}


Ruiz-Ruiz, Marcos Fernando; Noriega-Aranibar, María Teresa y

Pease-Dreibelbis, María Angélica

Brecha de género en la graduación de ingenieras industriales peruanas

Aguirre, J. C., y Jaramillo, L. G. (2015). El papel de la descripción en la investigación cualitativa. Cinta de Moebio, (53), 175-189. $\quad$ http://doi.org/10.4067/ $\underline{\mathrm{S} 0717-554 \mathrm{X} 2015000200006}$

Ames, P. (2006). Las brechas invisibles: Desafios para una equidad de género en la educación. Instituto de Estudios Peruanos - IEP.

Bandura, A. (1986). Social foundations of thought and action: A social cognitive theory. Prentice-Hall.

Bannikova, L. N., Baliasov, A. A., y Kemmet, E. V. (2018). Attraction and retention of women in engineering. In IEEE International Conference "Quality Management, Transport and Information Security, Information Technologies" (IT\&QM\&IS) (pp. 824827). IEEE. https://doi.org/10.1109/ ITMQIS.2018.8525043

Barnard, S., Powell, A., Bagilhole, B. M., y Dainty, A. (2010). Researching UK women professionals in SET: A critical review of current approaches. International Journal of Gender, Science and Technology, 2(3), 361381.

Barragán, C. A., González, A. D. C., y Ortiz, A. G. (2020). Competencias gerenciales: Una visión estudiantil desde la Pontificia Universidad Católica del Ecuador. Revista de Ciencias Sociales (Ve), XXVI(E-2), 113-126. https://doi. org/10.31876/rcs.v26i0.34117

Berkwits, M., y Inui, T. S. (1998). Making use of qualitative research techniques. Journal of General Internal Medicine, 13(3), 195-199. https://doi.org/10.1046/j.15251497.1998.00054.x

Blosser, E. (2017). Gender segregation across engineering majors: How engineering professors understand women's underrepresentation in undergraduate engineering. Engineering Studies, 9(1), 24-44. https://doi.org/10.1080/1 $\underline{9378629.2017 .1311902}$

Brawner, C. E., Camacho, M. M., Lord, S. M., Long, R. A., y Ohland, M. W. (2012). Women in industrial engineering: Stereotypes, persistence, and perspectives. Journal of Engineering Education, 101(2), 288-318. https:// doi.org/10.1002/j.2168-9830.2012. $\underline{\mathrm{tb} 00051 . \mathrm{x}}$

Castillo-SantaMaría, B., Ruiz-Nizama, J. L., Castillo-SantaMaría, I., y SaavedraRamírez, G. (2020). Educación y recursos económicos en mujeres del campo en Perú. Revista de Ciencias Sociales (Ve), XXVI(E-2), 81-93. $\quad$ https://doi.org/10.31876/rcs. v26i0.34115

Ceci, S. J., Ginther, D. K., Kahn, S., y Williams, W. M. (2014). Women in academic science: A changing landscape. Psychological Science in the Public Interest, 15(3), 75-141. https://doi. org/10.1177/1529100614541236

Chávez, V. A., Reyes, J. R., Carrillo, M. V., y Rodríguez, Á. F. (2020). Diferencias de género en unidades educativas rurales de Ecuador. Revista de Ciencias Sociales (Ve), XXVI(1), 203218. https://doi.org/10.31876/rcs. v26i1.31320

Cheryan, S., Master, A., y Meltzoff, A. N. (2015). Cultural stereotypes as gatekeepers: Increasing girls' interest in computer science and engineering by diversifying stereotypes. Frontiers in Psychology, 6(49), s/pp. http://doi. org/10.3389/fpsyg.2015.00049

Creswell, J. W. (Ed.) (2007). Qualitative inquiry and research design: Choosing among five approaches. Sage Publications.

Crosnoe, R., Riegle-Crumb, C., Field, S., Frank, K., y Muller, C. (2008). Peer group 
contexts of girls' and boys' academic experience. Child Development, 79(1), 139-155. http://doi.org/10.1111/ j.1467-8624.2007.01116.x

Dasgupta, N., y Stout, J. G. (2014). Girls and women in science, technology, engineering, and mathematics: STEMing the tide and broadening participation in STEM careers. Policy Insights from the Behavioral and Brain Sciences, 1(1), 21-29. http://doi. org/10.1177/2372732214549471

DiCicco-Bloom, B., y Crabtree, B. F. (2006). The qualitative research interview. Medical Education, 40(4), 314321. http://doi.org/10.1111/j.13652929.2006.02418.x

Downey, G. L., y Lucena, J. C. (2005). National identities in multinational worlds: Engineers and 'engineering cultures'. International Journal of Continuing Engineering Education and Lifelong Learning, 15(3-6), 253-260.

Dryburgh, H. (1999). Work hard, play hard: Women and professionalization in engineering - adapting to the culture. Gender \& Society, 13(5), 664-682. https://doi. org/10.1177/089124399013005006

Eccles, J. S. (2015). Gendered socialization of STEM interests in the family. International Journal of Gender, Science, and Technology, 7(2), 116132.

Faulkner, W. (2009a). Doing gender in engineering workplace cultures. I: Observations from the field. Engineering Studies, 1(1), 3-18. https:// doi.org/10.1080/19378620902721322

Faulkner, W. (2009b). Doing gender in engineering workplace cultures. II: Gender in/authenticity and the in/ visibility paradox. Engineering Studies, 1(3), 168-189. https://doi. org/10.1080/19378620903225059
Glaser, B. G., y Strauss, A. L. (2017). Discovery of grounded theory: Strategies for qualitative research. Routledge. https:// doi.org/10.4324/9780203793206

Goulden, M., Mason, M. A., y Frasch, K. (2011). Keeping women in the science pipeline. The ANNALS of the American Academy of Political and Social Science, 638(1), 141-162. https://doi. org/10.1177/0002716211416925

Guerrero, G., y Rojas, V. (2017). Young women and higher education in Peru: How does gender shape their educational trajectories? Gender and Education, 32(8), 1090-1108. http://doi.org/10.10 $\underline{80 / 09540253.2018 .1562055}$

Gunderson, E. A., Ramirez, G., Levine, S. C., y Beilock, S. L. (2011). The role of parents and teachers in the development of gender related math attitudes. Sex Roles, 66(3), 153-166. http://doi.org/10.1007/s11199-0119996-2

Hammersley, M. (2018). The dilemma of qualitative method. Routledge. https:// doi.org/10.4324/9781351037709

Herman, C., y Lewis, S. (2012). Entitled to a sustainable career? Motherhood in science, engineering, and technology. Journal of Social Issues, 68(4), 767789. https://doi.org/10.1111/j.1540$\underline{4560.2012 .01775 . x}$

Howe-Walsh, L., y Turnbull, S. (2016). Barriers to women leaders in academia: Tales from science and technology. Studies in Higher Education, 41(3), 415-428. https://doi.org/10.1080/03075079.201 4.929102

Howe-Walsh, L., Turnbull, S., Papavasileiou, E., y Bozionelos, N. (2016). The influence of motherhood on STEM women academics' perceptions of organizational support, mentoring and networking. Advancing Women in Leadership, 36, 54-63. 
Ruiz-Ruiz, Marcos Fernando; Noriega-Aranibar, María Teresa y

Pease-Dreibelbis, María Angélica

Brecha de género en la graduación de ingenieras industriales peruanas

Institute of Industrial \& Systems Engineers - IISE (2019). Origins of Institute of Industrial and Systems Engineers. https://www.iise.org/details. aspx?id=295

Leaper, C., Farkas, T., y Brown, C. S. (2012). Adolescent girls' experiences and gender-related beliefs in relation to their motivation in math/science and English. Journal of Youth and Adolescence, 41(3), 268-282. http:// doi.org/10.1007/s10964-011-9693-Z

Lerena, R. G., y Páez, A. C. (Eds.) (2019). Matilda y las mujeres en ingeniería en América Latina. Universidad FASTA Ediciones

Llanes, A., Cervantes, M. J., Peña, A. A., Cruz, J. (2020). Maternidad en legislación mexicana: Una visión desde los derechos laborales de la mujer. Revista de Ciencias Sociales (Ve), XXVI(1), 51-60. https://doi.org/10.31876/rcs. v26i1.31310

Martínez, K. I. (2018). Segregación laboral: El caso de las ingenieras mecánicas electricistas. Cuestiones de género: De la igualdad y la diferencia, (13), 89-105. http://doi.org/10.18002/ cg.v0i13.5402

Merriam, S. B., y Tisdell, E. J. (2015). Qualitative research: A guide to design and implementation. John Wiley \& Sons.

Motta, A., Keogh, S. C., Prada, E., NúnezCurto, A., Konda, K., Stillman, M., y Cáceres, C. M. (2017). De la normativa a la práctica: La política de educación sexual y su implementación en el Perú. Guttmacher Institute. https:// www.guttmacher.org/sites/default/ files/report pdf/de-la-normativa-a-lapractica-educacion-sexual-peru.pdf

Nazemi, S., Mortazavi, S., y Borjalilou, S. (2012). Investigating factors influencing women's inclination in adopting managerial positions

in Iranian higher education.

Interdisciplinary Journal of

Contemporary Research in Business, 4(7), 722-732.

Nguyen, T. L. H. (2013). Barriers to and facilitators of female Deans' career advancement in higher education: an exploratory study in Vietnam. Higher Education, 66(1), 123-138. https://doi. org/10.1007/s10734-012-9594-4

Oliveros, M. A., Cabrera, E., Valdez, B., y Schorr, M. (2016). La motivación de las mujeres por las carreras de ingeniería y tecnología. Entreciencias: Diálogos en la sociedad del conocimiento, 4(9), 89-96. http://dx.doi.org/10.21933/J. EDSC.2016.09.157

Olmedo, N., Sánchez, F., Salán, M. N., López, D., Perez, A., y López, M. (2018). Perfil de las ingenieras TIC versus el de otras mujeres STEM. En Actas de las XXIV Jornadas sobre Enseñanza Universitaria de la Informática, Vol. 3 (pp. 255-262). Asociación de Enseñantes Universitarios de la Informática (AENUI).

Pease, M. A. (1999). Lo que somos y lo que queremos ser. Jóvenes limeños construyendo identidad [Tesis de pregrado, Pontificia Universidad Católica del Perú]. https:// tesis.pucp.edu.pe/repositorio/ handle/20.500.12404/8082

Pease, M. A., Guillén, H., De La TorreBueno, S., Urbano, E., Aranibar, C., y Renjifo, F. (2019). Nuestra deuda con la adolescencia. Proyecto "Ser adolescente en el Perú" Convenio UNICEF-PUCP. https://www. unicef.org/peru/media/7136/file/ Nuestra $\% 20$ deuda $\% 20$ con $\% 201 \mathrm{a} \% 20$ adolescencia.pdf

Portocarrero, G. (1998). Ajuste de cuentas: Las clases medias en el trabajo de TEMPO. En G. Portocarrero (Coord.), 
Las clases medias: Entre la pretensión y la incertidumbre (pp. 9-37). SUR Casa de Estudios del Socialismo.

Regner, I., Steele, J. R., Ambady, N., ThinusBlanc, C., y Huguet, P. (2014). Our future scientists: A review of stereotype threat in girls from early elementary school to middle school. Revue Internationale de Psychologie Sociale, 27(3-4), 13-51.

Reinking, A., y Martin, B. (2018). The gender gap in STEM fields: Theories, movements, and ideas to engage girls in STEM. Journal of New Approaches in Educational Research, 7(2), 148-153. http://doi.org/10.7821/ naer.2018.7.271

Rodríguez, A. (2008). “Aquí hay que hacerse respetar". Mujeres entre tuercas y metales. Una mirada desde las mujeres de las facultades de Ingeniería de la Pontificia Universidad Católica del Perú. En M. Benavides (Ed.), Análisis de programas, procesos y resultados educativos en el Perú: Contribuciones empiricas para el debate (pp. 177207). GRADE, Grupo de Análisis para el Desarrollo.

Ruiz, P. (1997). Una aproximación al concepto de género. Cedoc.

Sarathchandra, D., Haltinner, K., Lichtenberg, N., y Tracy, H. (2018). "It's broader than just my work here: Gender variations in accounts of success among engineers in U.S. academia. Social Sciences, 7(3), 32. http://doi. org/10.3390/socsci7030032

Shapiro, J. R., y Williams, A. M. (2012). The role of stereotype threats in undermining girls' and women's performance and interest in STEM fields. Sex Roles, 66(3-4), 175-183. http://doi.org/10.1007/s11199-0110051-0

Sofaer, S. (1999). Qualitative methods: What are they and why use them? Health Services Research, 34(5Pt 2), 11011118.

Soilemezi, D., y Linceviciute, S. (2018). Synthesizing qualitative research: Reflections and lessons learnt by two new reviewers. International Journal of Qualitative Methods, 17(1). http:// doi.org/10.1177/1609406918768014

Starr, C. (2018). "I'm not a science nerd!": STEM stereotypes, identity, and motivation among undergraduate women. Psychology of Women Quarterly, 42(4), 489-503. https://doi. org/10.1177/0361684318793848

Steinke, J. (2017). Adolescent girls' STEM identity formation and media images of STEM professionals: Considering the influence of contextual cues. Frontiers in Psychology, 8, 716. http:// doi.org/10.3389/fpsyg.2017.00716

Stoet, G., y Geary, D. C. (2018). The gender-equality paradox in science, technology, engineering, and mathematics Education. Psychological Science, 29(4), 581-593. https://doi. org $/ 10.1177 / 0956797617741719$

Superintendencia Nacional de Educación Superior Universitaria del Perú - SUNEDU (2019). Información estadística de universidades. https:// www.sunedu.gob.pe/informacionestadistica-universidades-licenciadas/

Urbano, E. S. (2018). Lo que quiero ser: Toma de decisiones respecto a la ocupación futura en adolescentes de dos instituciones educativas de Lima [Tesis de pregrado, Pontificia Universidad Católica del Perú]. https://tesis.pucp.edu.pe/repositorio/ handle/20.500.12404/13659

Van Hoorn, J., Van Dijk, E., Meuwese, R., Rieffe, C., y Crone, E. A. (2016). Peer influence on prosocial behavior in adolescence. Journal of Research on 
Ruiz-Ruiz, Marcos Fernando; Noriega-Aranibar, María Teresa y

Pease-Dreibelbis, María Angélica

Brecha de género en la graduación de ingenieras industriales peruanas

Adolescence, 26(1), 90-100. http://doi. org/10.1111/jora. 12173

Williams, W. M., y Ceci, S. J. (2012). When scientists choose motherhood: A single factor goes a long way in explaining the dearth of women in math-intensive fields. How can we address it? American Scientist, 100(2), 138-145. https://doi.org/10.1511/2012.95.138

Yin, R. K. (2009). Case study research: Design and methods. Sage.

You, S. (2011). Peer influence and adolescents' school engagement. Procedia Social and Behavioral Sciences, 29, 829-835. http://doi.org/10.1016/j. sbspro.2011.11.311 